\title{
LA INTERVENCIÓN DEL BANCO MUNDIAL EN EL PROCESO DE REFORMA A LA EDUCACIÓN COLOMBIANA
}

GUILLERMO ANDRÉS DUQUE SILVA UNIVERSIDAD COOPERATIVA DE COLOMBIA

YULY ANDREA GARCÍA CASTILLO UNIVERSIDAD DEL VALLE

COLOMBIA

«Un país que destruye la Escuela Pública no lo hace nunca por dinero, porque falten recursos o su costo sea excesivo. Un país que desmonta la Educación, las Artes o las Culturas, está ya gobernado por aquellos que sólo tienen algo que perder con la difusión del saber». 


\title{
RESUMEN
}

\begin{abstract}
ste estudio analiza las políticas que buscan el ajuste lineal del sistema educativo colombiano a las demandas del mercado con el fin de seguir las directrices de los organismos internacionales, específicamente del Banco Mundial. Para lo cual se ha realizado un recorrido histórico de las políticas educativas más relevantes que sustentan la implementación de la evaluación por competencias en la educación colombiana, con la finalidad de reducir el proceso educativo a una preparación para el trabajo y el mundo laboral. El artículo se enfoca en mostrar que la intervención del BM ha transformado las obligaciones del Estado en cuanto a la prestación del servicio educativo, así como, la investigación, en los niveles de enseñanza superior de un derecho fundamental al nivel de un instrumento orientado en atender los problemas del sistema económico e industrial nacional.
\end{abstract}

Palabras clave: Política educativa colombiana, Sistema Educativo colombiano, Banco Mundial, Competencias, Investigación en Educación Superior.

\begin{abstract}
This study analyzes policies aimed linear fit Colombian educational system to market demands in order to follow the guidelines of international organizations such as the World Bank and IMF. For which there has been a historical tour of the most important education policies, which support the implementation of competency-based assessment in education today, with the aim of reducing the educational process to a preparation for work and the workplace. The article focuses on intervention show that the BM has transformed the State's obligations regarding the provision of educational services, as well as research, higher education levels of a fundamental right to the level of an instrument focused on meeting the problems of national economic and industrial system.
\end{abstract}

Keywords: Education Policy Colombian, Colombian Education System, World Bank, Skills, Higher Education Research.

Hay que reconocer que toda sociedad necesita formar personas para la labor idónea del trabajo, de no ser así sería imposible la supervivencia, y a la educación le corresponde contribuir con esto. Pero la labor del humanista, el pedagogo y el científico social es identificar el momento en que la inserción al mercado en la educación se invierte, al punto que la ley de la ofer- 
ta y demanda transgrede la esencia de la educación como un derecho fundamental. La actual promoción de una ideología de preparación para el mundo laboral mediante un saber hacer en contexto, casi no encuentra contradictores en un medio que ha internalizado el principal derrotero de la intervención de la economía en la educación: La formación por competencias.

La formación basada en competencias tiende a asumirse desde un pensamiento simple y hay un gran vacío en cuanto a su procedencia y real interés. A partir de esto, el presente artículo tiene como propósito realizar un recorrido histórico de las políticas educativas más relevantes, que sustentan la implementación de la evaluación por competencias en la educación actual.

Hasta los años setenta, según los programas antiguos de 1937 para la primaria, y en los de 1951 y 1962 para la secundaria, se debía evaluar por contenidos. A principios de los ochenta bajo el mandato del presidente Betancur (1982-1986), se realizaron algunas reformas en el sistema educativo con el fin de ofrecer recursos para la modernización, descentralización y planificación de la educación. De acuerdo con los diagnósticos que se realizaron de los gobiernos anteriores, la educación continuaba presentando problemas de calidad, cobertura y disponibilidad de recursos, sobre todo, la escuela rural. Ante esta situación y después de experimentar por varios años con los modelos de escuela unitaria, en la cual un maestro atiende simultáneamente cinco grados escolares, el Ministerio de Educación Nacional (MEN) adoptó el modelo de Escuela Nueva en 1976.

La Escuela Nueva se estableció como proyecto fundamental para la ampliación en la cobertura y la calidad de la educación en zonas rurales, recibiendo el apoyo de organizaciones internacionales y siendo punto de referencia de programas que se realizaron en América Latina durante los ochenta y noventa (Ramírez y Tellez, 2006). En ese momento, las voluntades nacionales para el mejoramiento de la educación rural son respaldadas por el Banco Mundial (BM), llevando a cabo un ambicioso proyecto para ofrecer una educación de alta calidad a las zonas más afectadas de Colombia. Con este proyecto el país sudamericano recibe el primer préstamo del Banco Mundial para financiar la educación.

Durante este periodo el BM interviene en la reforma educativa del país, bajo sus parámetros e intereses, "los logros del proyecto fueron adecuados en términos de mejoras en la calidad a pesar, como lo señala Duarte (1997), de algunas prácticas clientelistas en algunas regiones que sustituyeron los criterios técnicos”.

Seguido a esto, se inserta la ola de la tecnología educativa y el diseño instruccional, de corte conductista entonces hegemónica, la cual cambió el sistema evaluativo, y lo que anteriormente eran contenidos pasaron a ser objetivos generales, cada objetivo general se ramificó en numerosos objetivos específicos. Así con los programas de los decretos 1710 de 1963 para la primaria y el o80 de 1974 para la secundaria, propuestos por el MEN y consolidados con la asesoría de los Cuerpos de Paz, trascurrieron los setenta y los ochenta trabajando con objetivos específicos, hasta acostumbrarse a ellos. 
En 1982, se lleva a cabo el XII Congreso de la Federación Colombiana de Trabajadores de la Educación (FECODE), el cual orientó a los maestros colombianos hacia el desarrollo de un Movimiento Pedagógico que se dio a la tarea de analizar y debatir sobre aspectos fundamentales y determinantes de la educación pública: la calidad de la educación, el papel de la escuela en la sociedad colombiana, el papel del maestro, entre otros. Consecuencia y logro de este proceso fue la presentación al Congreso de la República y su posterior aprobación del proyecto de la Ley General de Educación (Bocanegra, s.f.p.).

La Constitución de 1991, define "la educación como un derecho fundamental y afirma que el Estado debe velar por la calidad de la educación”, impulsó, como en periodos anteriores, el proceso de descentralización de la administración pública y se consagró la educación como un derecho de la persona y un servicio público con función social. En el marco de la Nueva Constitución, la Ley 60 de 1993 definió las competencias y funciones de los tres niveles del Estado en los sectores de educación y salud y distribuyó el situado fiscal. Cabe recordar que en 1987, se inició el Plan para la Universalización de la Educación Básica Primaria y en 1992 el Programa de Ampliación de la Cobertura y Mejoramiento de la Calidad de la Educación Secundaria (Paces), ambos con cofinanciación del Banco Mundial. Este último programa, entrega financiación a la demanda de educación bajo condiciones de rendimiento académico y necesidades financieras, de tal forma que los estudiantes pobres puedan realizar sus estudios secundarios (Ramírez y Tellez, 2006, p. 65).

A partir de este momento, aparecen nuevas disposiciones legales, que rigen el sector educativo en función de la calidad. Durante la naciente década del noventa el BM se expresa enfocando los créditos a la educación secundaria y técnica, y la educación primaria, (Ver figura 1) estableciendo las condiciones sobre el sentido de la educación, principalmente, como un instrumento y no como un derecho universal.

\section{Créditos del Banco Mundial para la educación en ALC por subsector}

Ejercicios 92 a 96

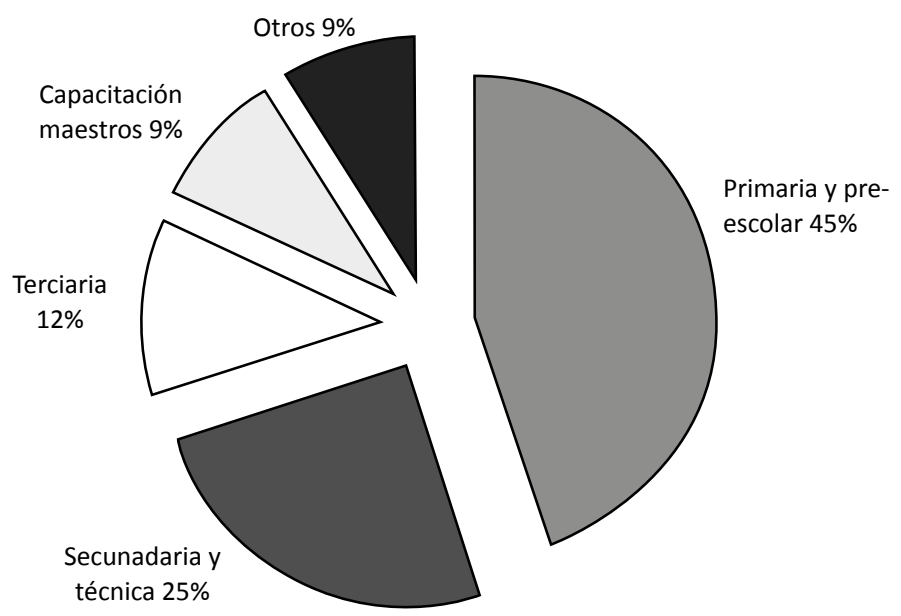

Fuente: Banco Mundial (2000) 
El Banco Mundial define que el Estado no es el único responsable de la educación en América Latina, y explica el papel preponderante que tiene la inversión privada en este sector de la economía del país. En este sentido, los años noventa se dinamizaron bajo una premisa, según, el BM (2000):

“Aunque el papel del Estado continúa siendo el fomento de la educación básica para todos, ya no es el único responsable de impartir la educación propiamente dicha. Se debe limitar la participación del gobierno a aquellas actividades que el sector privado no puede llevar a cabo eficazmente" (p. 37).

Simultáneamente, y después de un complejo proceso de negociación con el magisterio, se expide la Ley 115 de 1994 o "Ley General de Educación”, la cual establece los principios para la dirección, administración y financiación del sistema educativo, asigna un mayor rol a los departamentos en la administración de los recursos, transfiere los Fondos Educativos Regionales a los Departamentos y crea nuevos esquemas para la evaluación de la calidad de la educación, con el fin de estandarizar la educación bajo las condiciones políticas del BMํ․ Nace también el Decreto 1860 de 1994, el cual reglamenta el proceso pedagógico y la organización de la institución educativa, a través del Proyecto Educativo Institucional, el gobierno escolar y las normas de evaluación y promoción. Durante este periodo el valor de los créditos educativos se triplica en la región Latinoamericana, especialmente en Colombia ampliando la relación de dependencia con el BM.

Se condiciona entonces la promulgación de la nueva ley general de educación, la Ley 115, resultado de la fuerza sindical docente, los comités de expertos y los congresistas que asumieron la posición del BM. Finalmente, como lo afirma la entidad bancaria en mención, en el diálogo con los diversos gobiernos la voluntad del organismo financiero se terminó imponiendo en nombre del desarrollo de la región. En su informe de fin de siglo, el BM, que en los noventa como en toda reforma a la educación, se caracterizó por "contraponer las necesidades educacionales de cada país con el compromiso del Banco de propiciar cambios necesarios de política y su capacidad de instaurar programas y políticas" (BID 2000, p. 13). 
Figura 2.

Volúmen de Créditos del Banco Mundial para la educación en ALC

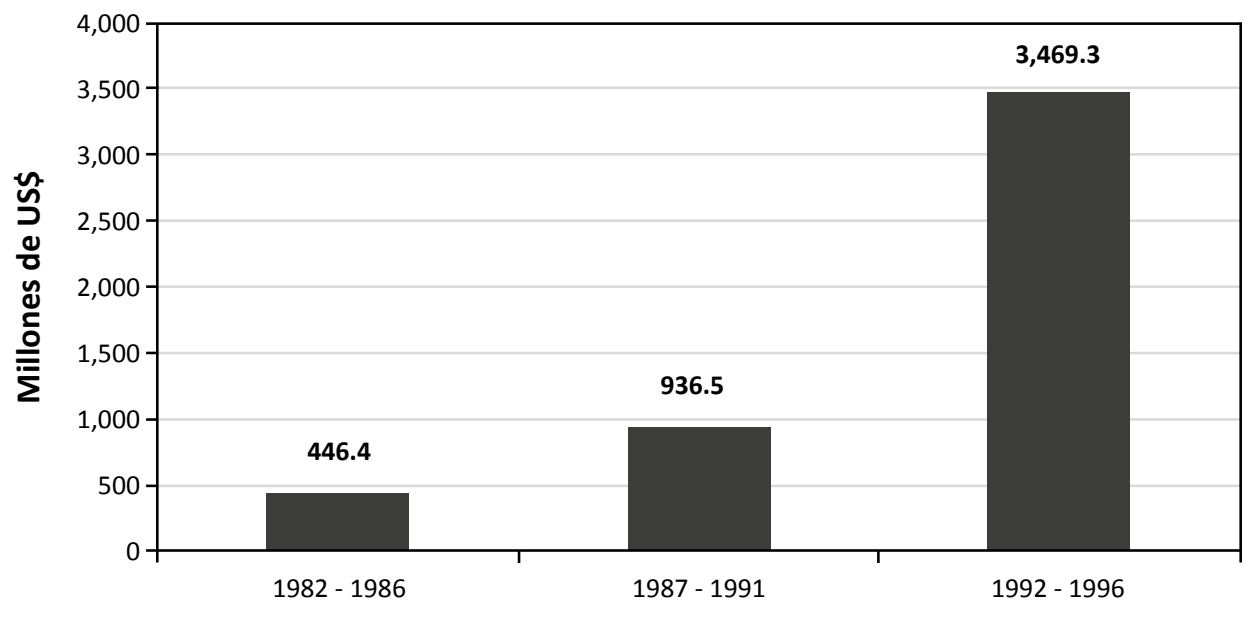

Fuente: Banco Mundial (2000)

Después de la Ley General de Educación nace la Resolución 2343 de 1996, en la cual se establece una lista de indicadores de logros para ciertos grupos de grados. Sin embargo, este proceso de evaluación se modifica, porque el Instituto Colombiano para la Evaluación de la Educación (ICFES) y la Secretaría de Educación de Bogotá, decidieron que era mejor diseñar una evaluación por competencias, y por otra parte, al Plan Nacional de Desarrollo del gobierno de Andrés Pastrana Arango, quien había decidido que la evaluación debía ser por estándares de competencia, los cuales buscaban la uniformidad (Vasco, 2003). Generando confusiones entre los objetivos específicos, los logros y los indicadores de logros. Impulsado en primera instancia por el seguido a esta decisión, se establece en el mismo año, la Resolución No. 2707 del 26 de Junio de 1996, mediante la cual se definen los criterios generales para la evaluación institucional y de docentes estatales ligada al otorgamiento de incentivos de calidad (UNESCO).

En 1998, la Alcaldía de Bogotá al mando de Enrique Peñalosa, la ministra de Educación María Cecilia Vélez ${ }^{2}$, participa activamente en los acuerdos con el Banco Mundial. El cual se manifiesta en la Cumbre de las Américas II, de modo abierto, que su objetivo es transformar a profundidad el sistema educativo latinoamericano, desde el modelo de gestión y distribución de recursos hasta las prácticas pedagógicas. Afirma el Banco que:

"Los métodos pedagógicos y currículos actuales tienden a reflejar métodos y actitudes que ya no tienen vigencia, que conservan la rica herencia social y cultural 
del pasado pero no satisfacen los requisitos urgentes del presente ni las necesidades apremiantes del futuro. Esta falta de aplicabilidad es particularmente problemática dado el contexto económico, las condiciones del mercado laboral, el desplazamiento hacia la democracia, y el consenso regional expresado en la Cumbre de las Américas II acerca de la necesidad de aumentar y mejorar el capital humano de la región” (BM: 2000, p. 55).

En cumplimiento de las directrices del Banco, surgen así las propuestas de "reorganización institucional y optimización de la capacidad instalada” y se lanza el "Plan Estratégico" del Ministerio de Educación y el "Plan de reorganización del sector educativo" (PRESE), los cuales fueron coadyuvados por el Ministerio de Hacienda y el Departamento Nacional de Planeación en el año 2000.

Mediante la justificación de "racionalizar" y "aprovechar el máximo de los recursos", se dio inició al desarrollo de la relación maestro - alumno, al precio de un verdadero hacinamiento en las aulas escolares, se ampliaron los convenios de desempeño con los departamentos bajo el principio del "subsidio a la demanda", se incrementó la cobertura, se fusionaron y cerraron instituciones, se suprimieron las direcciones de escuelas y se eliminó la repetición de grados. Con esto la mayoría de las prioridades demandadas por el BM fueron atendidas.

Por ejemplo, en el municipio de Antioquia, desde el gobierno departamental de Uribe, se dio el paso a los contratos y convenios privatizadores. Sin embargo, pocos municipios y departamentos contrataron maestros para suplir con recursos propios el déficit educativo, entrando en crisis. Al final, los resultados fueron claros: se crearon 600 mil cupos pero 650 mil niños desertaron del sistema escolar (Atehortúa, 2006).

Sin embargo, a fines de los años noventa, el BM extremó sus posturas y demostró la importancia de las tendencias y desafíos que, en su opinión, motivaban la reforma del sector educativo en América Latina con lo que, desde luego, condicionó sus préstamos. El evento se realiza en el marco de en una reunión de ministros de educación y líderes del sector privado de América Latina y el Caribe convocada por el Presidente del Banco Mundial, James Wolfensohn, y celebrada en junio de 1998 en la sede del Banco en Washington, D.C. La postura del Banco para la década naciente se puede se resumir en cuatro ejes:

* Los rápidos cambios económicos y tecnológicos están exigiendo que los gobiernos de la región inviertan en las habilidades y la educación de la fuerza laboral.

* El mayor acceso a la educación es la clave para reducir la persistente pobreza y desigualdad social en los países de la región. 
* Para que las nuevas democracias de la región prosperen, deben contar con la amplia participación de una ciudadanía educada e informada.

* Los cambios en el papel y la organización del Estado significan que el gobierno central ya no es el único responsable de prestar servicios educativos, sino que cada vez más comparte esa función con los gobiernos locales, las comunidades, las familias, las personas individuales y el sector privado (World Bank, 1999).

En respuesta a estas condiciones, surge la casi inmediata acción del Estado colombiano expresada a través del “Acto Legislativo o1" y la "Ley 715 o de Transferencias”, con los cuales se reformaron los artículos 356 y 367 de la Constitución Política Nacional, se eliminó el situado fiscal ya congelado y reducido desde el gobierno de Ernesto Samper (1994-1998), y se impuso un nuevo esquema para la asignación de recursos en la educación pública: el Sistema General de Participaciones, traducido en la municipalización y plantelización de la financiación educativa, el subsidio a la demanda (Atehortúa, 2012).

El 24 de julio de 2002 se promulga el Decreto 1528, con el cual se reglamentan parcialmente los artículos 23 y 27 de la Ley 715 y se posibilita la contratación con entidades privadas entregando incluso establecimientos del Estado. Se trata de un componente, que obedece nuevamente las recomendaciones dadas por el Banco. A esto se sumó un nuevo Estatuto Docente y un nuevo sistema de inspección y vigilancia impuesto al final de la administración de Pastrana. Este último, plasmado en el Decreto 1283 de 2002, privilegió a la empresa privada educativa y persiguió, desde su esquema tecnocrático de evaluación de desempeño y competencias, a las instituciones y docentes públicos.

En el mismo año se instituye el Decreto No. 230 de 2002, el cual reglamenta las disposiciones de la Ley General de Educación, relacionadas con la inspección y vigilancia, los criterios para la evaluación del rendimiento escolar de los educandos y su promoción a niveles superiores, así como las disposiciones de la ley 715 de 2001 de Recursos y Competencias, en el que le corresponde a la Nación establecer normas técnicas curriculares y pedagógicas para los niveles de educación preescolar, básica y media, sin perjuicio de la autonomía escolar que tienen los establecimientos educativos y de la especificidad de tipo regional, y quien define, diseña y establece instrumentos y mecanismos para el mejoramiento de la calidad de la educación.

Estas políticas y estrategias se afirmaron aún más con la continuidad de Cecilia María Vélez como Secretaria de Educación en la administración de Antanas Mockus (2001-2003) y con su presencia de ocho años en el Ministerio de Educación bajo el gobierno de Álvaro Uribe Vélez (2002-2010).

A partir del 2002 empiezan a gestionarse los Planes de Desarrollo, en el que la educación juega un papel importante. En las “Bases del Plan Nacional de Desarrollo 2002-2006”, 
la Educación se definió como "factor esencial del desarrollo humano, social y económico y un instrumento fundamental para la construcción de equidad social” (DNP, 2002, p. 122). Desaparecen algunas ofertas que para entonces Álvaro Uribe Vélez en su candidatura había declarado; como la capacitación y la investigación científica, sin embargo, éstas se borraron como estrategias. Al lado de la cobertura, la calidad y la pertinencia, apareció una nueva que era, como afirma Atehortúa (2006), la más vieja de todas: "la eficiencia".

El primer mecanismo para el mejoramiento de la calidad de la educación que se propone en este plan, consiste en la definición de estándares de calidad para todos los niveles de educación, con el propósito de unificar los objetivos del sistema educativo. Igualmente se promueve el desarrollo de competencias básicas para alcanzar desempeños satisfactorios en su actividad laboral (DNP, 2002, p. 179).

Según el documento, a pesar del progreso obtenido durante los últimos años, las estadísticas nacionales mostraban un porcentaje considerable de la población en edad escolar por fuera del sistema educativo, y una inequidad pronunciada en posibilidades de acceso y permanencia. La calidad era deficiente y en un acelerado detrimento, primordialmente en los grados de formación básica y media. Además, el proyecto institucional educativo no producía incentivos a la eficiencia y limitaba el avance en la expansión de cobertura y mejoramiento de la calidad.

Durante el gobierno de Uribe, el compromiso de la prestación del "servicio educativo" se enfocó en un método efectista, es decir centrado en la cobertura y la eficiencia financiera del sistema, para ello buscó dejar de lado lentamente las obligaciones del Estado y ceder a las instituciones y a los particulares la obligación de autofinanciar la educación. La autonomía escolar se planteó como "autonomía real” de carácter financiero y de manejo de personal; es decir, autofinanciación.

Por lo cual la Educación se entendía, como afirma Atehortúa (2012, p. 9) como:

"factor esencial del desarrollo humano, social y económico y un instrumento fundamental para la construcción de equidad social, revela una visión utilitarista y reemplaza la definición de Educación como derecho, establecida en nuestra Constitución Nacional en el Título II, Artículos 44, 45 y 67: "la educación es un derecho fundamental de niños y jóvenes que debe ser asistido y garantizado por el Estado; un derecho de la persona y un servicio público con función social que se ofrecerá en forma obligatoria entre los cinco y quince años de edad, y gratuita en las instituciones del Estado”.

El BM expresa un mensaje claro a los gobiernos latinoamericanos frente a la colaboración privada, "la participación del sector privado no sólo aumenta el número efectivo de plazas escolares, sino que también mejora la calidad al fomentar la competencia por fondos públicos entre 
los proveedores públicos y privados” (BM, 2000, p. 35). Dicha participación se combina con el principio de la descentralización administrativa que les otorga a los gobiernos locales autonomía en la asignación de contratos y concesiones con privados para la prestación del servicio educativo. Así, una casa adaptada para ser colegio podía recibir millones al año por alumno mientras que se comprometiera a brindar el servicio de educación en las zonas donde no habían escuelas construidas. Este segundo programa de ampliación de cobertura se sustentó en las mismas recomendaciones del BM, para el cual "La participación del sector privado no sólo aumenta el número efectivo de plazas escolares, sino que también mejora la calidad al fomentar la competencia por fondos públicos entre los proveedores públicos y privados" (BM, 2000, p. 35).

La puesta en marcha de este programa desató uno de los más graves escándalos de corrupción que ha tenido el país. Entre el 2004 y el 2012 en el Valle del Cauca, sólo por mencionar uno de los treinta y dos Departamentos del país, se ha descubierto que más de 40 mil niños que no existían y eran pagados por el Estado a operadores privados, "los niños de papel”, como llama la prensa a este fenómeno le ha costado a la Nación unos 400 mil millones de pesos robados entre ediles locales y una nueva modalidad de testaferros de la ampliación de cobertura ${ }^{3}$.

Durante el segundo gobierno Uribe, la llamada "Revolución educativa" hallaría un nuevo aire, el cual se manifiesta en la redacción del Plan Nacional de Desarrollo (2006-2010) que retorna a un enfoque utilitarista, con el que se define abiertamente la educación como un "instrumento para lograr la inclusión social por características diferentes a las que tienen que ver con su nivel socioeconómico" (DNP, 2007, p. 150). Se instaura el Decreto No. 1767 de 2006 el cual define el sistema de información de educación superior, sus objetivos tanto generales como específicos, y las condiciones de calidad con las que debe contar la información reportada por las instituciones de educación superior al Ministerio de Educación (UNESCO, s.f.p.).

Más adelante, el Decreto No. 3433 de 2008 reglamenta la expedición de licencias de funcionamiento de los establecimientos no oficiales interesados en prestar el servicio educativo. Con esto se busca ejercer un mayor control sobre la totalidad de los establecimientos educativos (oficiales y no oficiales), contribuyendo de esta manera "al mejoramiento de la calidad educativa vía un mayor control de las condiciones iniciales, de enseñanza y de requisitos de los establecimientos educativos no oficiales".

En el 2009, a través del Decreto 1290, se reglamenta la evaluación del aprendizaje basado en los estándares básicos de calidad. De igual manera se establece la escala de valoración mediante desempeños, "la denominación Desempeño Básico se entiende como la superación de los desempeños necesarios en relación con las áreas obligatorias y fundamentales, teniendo como referente los estándares básicos, las orientaciones y lineamientos expedidos por el Ministerio de Educación Nacional y lo establecido en el proyecto educativo institucional” 4 .

\footnotetext{
${ }^{3}$ Al respecto ver: Diario de Occidente, 15 de abril de 2013. Disponible en:

http://www.occidente.co/polemica-por-ampliacion-de-cobertura-educativa-2/
}

${ }^{4}$ Decreto 1290, Art. 5. 
El término Calidad toma protagonismo sobre la base de la Ley 715 de 2001 y en el marco del Decreto 5012 de 2009, el cual establece los compromisos del Ministerio de Educación Nacional ${ }^{5}$.

En el Plan Nacional de Desarrollo (2010-2014) propuesto por el Presidente Juan Manuel Santos se insiste en que la educación "es el instrumento más poderoso para reducir la pobreza y el camino más efectivo para alcanzar la prosperidad" (DNP, 2011). Las concurrencias saltan a la vista. Pero no solo en la idea. La visión utilitarista de la Educación trae consigo una mirada precisa en las estrategias definidas al nivel gubernamental, tanto nacional como local.

En cada uno de los Planes y Programas el papel estratégico de la educación se ubicó en el aumento de las capacidades y destrezas personales, léase competencias, para el empleo y la productividad. Así lo expresa abiertamente el Plan 2006-2010: la "adquisición de conocimientos, competencias y habilidades productivas" es requisito para "contribuir al crecimiento e incrementar la productividad del empleo" (DNP, 2007, p. 152). Podría inferirse, con ingenuidad, que ese tipo de formación no es formación, es capacitación y se limita, por tanto, a las cualidades y destrezas que el individuo necesita para defenderse en el caos feroz del capital; para ingresar con relativo éxito pero desventurado reconocimiento al mercado laboral y "aumentar así la productividad" (Atehortúa, 2012).

La realidad es que la planeación académica hoy en día se basa más en desempeños intermedios y terminales que en áreas de conocimiento y temáticas, las estrategias didácticas enseñan y ponen a prueba los desempeños de los estudiantes ante problemas que resolver, retos que enfrentar, contingencias por manejar y alternativas por construir. Entonces, la evaluación mide y valora el nivel de competencia del desempeño estudiantil más que el dominio cognitivo que haya en temas y contenidos, la clave está ahora en el desempeño inicial del estudiante para llevarlo por una trayectoria formativa hacia los desempeños terminales expresados en el perfil del egresado del modelo institucional, un arquetipo que de antemano debe ser coherente con las exigencias del mercado laboral, esto es, el enfoque de competencias instalado a plenitud en la educación básica y media colombiana.

Con respecto al nivel académico universitario, el Plan de Desarrollo 2006-2010 planteó alianzas estratégicas entre instituciones de educación superior, instituciones con programas de educación media técnica, el sector productivo, los gobiernos regionales y locales, el SENA ${ }^{6}$, y centros de innovación, productividad y desarrollo tecnológico, con el fin de ampliar la oferta y crecer en cobertura.

En el caso del SENA, los programas abiertos se han realizado sobre la base de ciclos disciplinares dirigidos al desarrollo de competencias y acordes con los requerimientos del sector productivo. El problema con ello es, como afirma Atehortúa (2012, p.16) que:

\footnotetext{
$\overline{{ }^{5} \text { Sólo por mencionar un dato cuantitativo sobre este decreto; se evidencia que en menos de siete párrafos se puede contar la }}$ palabra calidad 12 veces.

${ }^{4}$ Servicio Nacional de Aprendizaje, principal referente en el hemisferio occidental de un sistema de formación técnica y tecnológica laboral.
} 
No existe una visión de conjunto curricular; no existe un proyecto de formación dirigida, ni un plan de estudios concreto; no impera un sistema progresivo y dinámico sobre la formación y no se obtiene la naturaleza continua de la educación formal. Dicho en otras palabras, se aplica la perspectiva de la capacitación para el empleo en reemplazo de la formación profesional con integralidad.

En el 2011, con respecto a la educación privada, en el Congreso de la República se da curso al "Proyecto de ley por el cual se organiza el Sistema Nacional de Educación Superior y se regula la prestación del servicio público de la Educación Superior" más conocido como "Reforma a la Ley 30 de Educación Superior", y que finalmente fue retirado por la presión del Movimiento Estudiantil. Este proyecto tampoco escondió sus procedencias. La expresión de Educación como derecho fue añadida al final, para demostrar cambios "debido a las reuniones con los estudiantes", pero el espíritu mercantilista y utilitarista de la educación como instrumento, continuó ileso. El término "competencias" apareció 23 veces a lo largo de un texto que descartaba toda posibilidad de autonomía universitaria frente a los fines de la educación profesional.

En tal propuesta de reforma a la Ley 30 de Educación Superior se establecía que para acceder a la Educación Superior se requiriera de las instituciones (Art. 7); el reconocimiento de las cualidades educativas, es decir, que estuviesen legalmente constituidas y autorizadas por el Ministerio de Educación Nacional. Girando en torno a las competencias laborales (Arts. 125, 133), formando en competencias como objetivo central de los programas técnicos, tecnológicos, profesionales y de posgrado (Arts. 60, 61, 62, 64 y 65 en donde aparecía tres veces, Art. 71 y 72); debía ser también el propósito de los doctorados y sus investigaciones (Art. 66), requisito del título (Art. 67), el cual debía hacerse constar en el diploma. Y lo que es más inadmisible:

Artículo 67, Parágrafo: Adjunto al diploma, las Instituciones de Educación Superior expedirán un documento suplemento, que contendrá información relativa al perfil y competencias desarrolladas en el programa, según reglamentación que expida el Gobierno Nacional (Atehortúa, 2012, p. 13).

¿En qué lugar quedaba la libertad de cátedra, el PEI y la autonomía universitaria? Las competencias, certificadas por medio de un diploma, se convertirían en la etiqueta del profesional.

De igual manera, las competencias en la "Propuesta de Reforma" serían la razón de ser de los exámenes de Estado (Art. 68), el eje de todo currículo (Art. 73), la base fundamental para la evaluación de calidad y su acreditación (Art. 92) y la pieza básica para la articulación de los niveles formativos (Art. 115).

De igual forma que con la educación superior, el presente gobierno se ha empeñado en dar cumplimiento a las indicaciones del BM frente a la formación para el sector productivo, lo hace con la Ley 1286 del 23 de Enero de 2009, con la que se reorienta la investigación en el

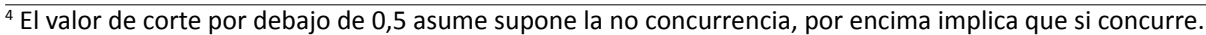


país, dando un viraje hacia las demandas del sector productivo, hoy por hoy eje de las acciones del Departamento Administrativo de Ciencia, Tecnología e Innovación, COLCIENCIAS7.

Actualmente, el Departamento de Filosofía de la Universidad del Valle lleva a cabo un estudio sobre la nueva Ley 1286 de 2009, ordenamiento que busca, entre otras cosas, como lo expresa el profesor William Gonzáles (2012), el beneficio de una “aristocracia de lo miserable”, que subasta el dinero público para la investigación en el sector privado, en la innovación para el sector productivo y en Planes de Desarrollo Nacionales y Regionales. La nueva Ley tiene como pretensión transformar a Colciencias en Departamento Administrativo y fortalecer el Sistema Nacional de Ciencia, Tecnología e Innovación en Colombia, para lograr un modelo productivo, "sustentado en la ciencia, la tecnología y la innovación, para darle valor agregado a los productos y servicios de nuestra economía y propiciar el desarrollo productivo y una nueva industria nacional".

La Ley 1286 define y orienta líneas temáticas prioritarias y operativas del Sistema Nacional de Ciencias, Tecnología e Innovación, para lo cual podrá modificar, suprimir o fusionar los Programas Nacionales de Ciencia, Tecnología e Innovación; crear nuevas estructuras sobre las diferentes áreas del conocimiento; definir su nombre, composición y funciones; dictar las reglas para su organización y diseñar las pautas para su incorporación en los planes de las entidades vinculadas con su ejecución." (Gonzáles, 2012, p. 8). Es decir, se centraliza el poder y la Universidad queda excluida de cualquier decisión o participación.

¿Pero qué dice esta Ley acerca de las Ciencias Sociales, la cual se supone que para Colciencias incluye a las Ciencias Humanas? Lamentablemente, no hay buenas noticias para este campo del conocimiento en materia de investigación. En su Capítulo IV la mencionada Ley, establece que "las Ciencias Sociales serán objeto específico de la investigación científica y recibirán apoyo directo para su realización”. El apoyo señalaba más una exclusión que una inclusión en el gran sistema de investigación. En la medida que, por ejemplo, las becas de postgrados para las ciencias humanas y sociales fueron eliminadas por Colciencias para el 2013, situación que pasó desapercibida por muchas instituciones. Simplemente por el hecho de que:

Muchos de los que no creen en una Universidad como Facultad de juicio crítico de la sociedad, tampoco creen en las ciencias sociales y humanas. No somos un área estratégica, es decir un buen negocio. Ellos tienen una visión empresarial, productiva (en el sentido de productos vendibles) y comercial de la universidad. (Gonzáles, 2012). 
La Ley 1286, revierte todo el modelo de decisión sobre la investigación en educación, y las decisiones de lo que hay que hacer ya no son tomadas por los grandes investigadores, sino por políticos de turno y gamonales del poder regional que en nombre de las regalías venden al mejor postor los recursos que antes se asignaban bajo estrictos criterios medianamente transparentes. Con la Ley 1286 la financiación estatal directa es casi inexistente. La tarea de esta nueva Ley es desmantelar la investigación en ciencias humanas, a través de un modelo productivo y una disminución en la financiación de la investigación (Gonzáles, 2012, p. 11).

Lamentablemente, Colciencias dirige la investigación hacia el hacer y no hacia al conocimiento, debido a que el interés en la educación sigue siendo instrumentalista, dando mayor importancia a la industria que al conocimiento, y como lo plantea el profesor Gonzáles (2012, p. 15) "muchos de nuestros investigadores se han robotizado, y han renunciado en nuestra época a ser nuevos Leonardo de Vinci, ya que están constreñidos por leyes como la 1286, a tratar al otro a través de su investigación como un cliente y no como seres humanos con necesidades, opiniones y problemas”.

En ese orden de ideas, detrás de esta nueva ley se observa una noción sesgada, reduccionista y arbitraria de lo que realmente es la ciencia, la tecnología y por supuesto la investigación. La investigación se encuentra en una crisis en la que es incapaz de entender, por fuera del sistema económico, los cambios actuales del conocimientos y las verdaderas necesidades de las comunidades tanto científicas como sociales (Gonzáles, 2012).

La Universidad asumió que la literatura científica se convirtiera en escrito de los que tienen el poder y de que son ellos los que construyen los modelos a seguir, además de sujetarse a las imposiciones dadas por políticas de investigación no siempre discutidas, como la última propuesta de medición, la cual dejan por fuera a aquellos que no se adaptan al régimen investigativo imperante (Gonzáles, 2012, p. 25). Así, son excluidos los practicantes de campo en humanidades, estudio de "casos" de filosofía, psicología, sociología, y aún los de producción literaria, artística y poética; todo con el fin de favorecer en nuestro país a la industria de grandes teorías y aplicaciones productivas y comerciales que mueve a la Ciencia, la Innovación y la Tecnología.

Este proceso sistemático de intervención del BM en la construcción de la agenda educativa colombiana puede sintetizarse en la Tabla 1, en la que se muestra la relación directa entre economía y educación. A través de la legislación, principalmente, leyes, decretos o directivas ministeriales, las cuáles impactan directamente en la instrumentalización, privatización y tercerización de la educación del país. 
Tabla 1

Síntesis histórica de la intervención del BM en la Reforma Educativa colombiana

\begin{tabular}{|c|c|c|}
\hline RECOMENDACIÓN DEL BANCO MUNDIAL & LEY/ DECRETO/ PLAN & EFECTOS DE SU APLICACIÓN \\
\hline $\begin{array}{l}\text { Los niveles gubernamentales en los que están reca- } \\
\text { yendo estas responsabilidades necesitarán mejorar } \\
\text { sus capacidades de gestión para que logren satisfa- } \\
\text { cer las necesidades de acceso y calidad. }\end{array}$ & $\begin{array}{l}\text { Constitución de } 1991 . \\
\text { Decreto } 1290 \text { de } 2008 . \\
\text { Ley } 715 \text { de } 2001 \text { y Acto } \\
\text { legislativo } 01 .\end{array}$ & $\begin{array}{l}\text { La cual define "la educación como un de- } \\
\text { recho fundamental y afirma que el Estado } \\
\text { debe velar por la calidad de la educación". } \\
\text { Reglamenta la evaluación del aprendiza- } \\
\text { je basado en los estándares básicos de } \\
\text { calidad. } \\
\text { Se eliminó el situado fiscal ya congelado } \\
\text { y reducido desde el gobierno de Ernesto } \\
\text { Samper, y se impuso un nuevo esquema } \\
\text { para la asignación de recursos en la edu- } \\
\text { cación pública: el sistema General de Par- } \\
\text { ticipaciones, traducido como la municipa- } \\
\text { lización y plantelización de la financiación } \\
\text { educativa, el subsidio a la demanda. } \\
\text { Le corresponde a la Nación establecer nor- } \\
\text { mas técnicas curriculares y pedagógicas } \\
\text { para los niveles de educación preescolar, } \\
\text { básica y media, sin perjuicio de la autono- } \\
\text { mía escolar que tienen los establecimien- } \\
\text { tos educativos y de la especificidad de tipo } \\
\text { regional, y quien define, diseña y establece } \\
\text { instrumentos y mecanismos para el mejo- } \\
\text { ramiento de la calidad de la educación. }\end{array}$ \\
\hline $\begin{array}{l}\text { En la Cumbre de las Américas de } 1998 \text {, los gobier- } \\
\text { nos de ALC dieron a la educación una alta priori- } \\
\text { dad como un medio de lograr la "competitividad } \\
\text { y productividad requeridas por las economías mo- } \\
\text { dernas", y de permitir que sus poblaciones "contri- } \\
\text { buyan como ciudadanos valiosos a sus sociedades } \\
\text { respectivas" (Cumbre de las Américas II, 1998). }\end{array}$ & $\begin{array}{l}\text { Ley } 60 \text { de } 1993 . \\
\text { Plan Nacional de } \\
\text { Desarrollo "2002-2006". }\end{array}$ & $\begin{array}{l}\text { Definió las competencias y funciones de } \\
\text { los tres niveles del Estado en los secto- } \\
\text { res de educación y salud y distribuyó el } \\
\text { situado fiscal. } \\
\text { Consiste en la definición de estándares de } \\
\text { calidad para todos los niveles de educa- } \\
\text { ción, con el propósito de unificar los obje- } \\
\text { tivos del sistema educativo. Igualmente se } \\
\text { promueve el desarrollo de competencias } \\
\text { básicas para alcanzar desempeños satis- } \\
\text { factorios en su actividad laboral. }\end{array}$ \\
\hline $\begin{array}{l}\text { Los cambios en el papel y la organización del es- } \\
\text { tado significan que el gobierno central ya no es el } \\
\text { único responsable de prestar servicios educativos, } \\
\text { sino que cada vez más comparte esa función con los } \\
\text { gobiernos locales, las comunidades, las familias, las } \\
\text { personas individuales y el sector privado. }\end{array}$ & $\begin{array}{l}\text { Ley } 115 \text { de } 1994 \text { o "Ley } \\
\text { General de Educación". }\end{array}$ & $\begin{array}{l}\text { La cual establece los principios para la } \\
\text { dirección, administración y financiación } \\
\text { del sistema educativo, asigna un mayor } \\
\text { rol a los departamentos en la administra- } \\
\text { ción de los recursos, transfiere los Fon- } \\
\text { dos Educativos Regionales a los departa- } \\
\text { mentos y crea nuevos esquemas para la } \\
\text { evaluación de la calidad de la educación, } \\
\text { con el fin de estandarizar la educación. }\end{array}$ \\
\hline
\end{tabular}




\begin{tabular}{|c|c|c|}
\hline RECOMENDACIÓN DEL BANCO MUNDIAL & LEY/ DECRETO/ PLAN & EFECTOS DE SU APLICACIÓN \\
\hline $\begin{array}{l}\text { El mayor acceso a la educación es la clave para re- } \\
\text { ducir la persistente pobreza y desigualdad social en } \\
\text { los países de la región. }\end{array}$ & $\begin{array}{l}1992 \text { el Programa de Am- } \\
\text { pliación de la Cobertura } \\
\text { y Mejoramiento de la Ca- } \\
\text { lidad de la Educación Se- } \\
\text { cundaria (Paces) } \\
\\
\text { "Plan de reorganización del } \\
\text { sector educativo" (PRESE) } \\
2000 .\end{array}$ & $\begin{array}{l}\text { Se entrega financiación a la demanda de } \\
\text { educación bajo condiciones de rendimien- } \\
\text { to académico y necesidades financieras, de } \\
\text { tal forma que los estudiantes pobres pue- } \\
\text { dan realizar sus estudios secundarios } \\
\text { Se dio inició al desarrollo de la relación } \\
\text { maestro - alumno, al precio de un ver- } \\
\text { dadero hacinamiento en las aulas esco- } \\
\text { lares, se ampliaron los convenios de des- } \\
\text { empeño con los departamentos bajo el } \\
\text { principio del "subsidio a la demanda", se } \\
\text { incrementó la cobertura, se fusionaron } \\
\text { y cerraron instituciones, se suprimieron } \\
\text { las direcciones de escuelas y se eliminó } \\
\text { la repetición de grados. }\end{array}$ \\
\hline $\begin{array}{l}\text { Diversificar y reformar la educación terciaria para } \\
\text { mejorar la calidad y eficiencia, mejorar el acceso } \\
\text { para los dos quintiles de menores ingresos, y forta- } \\
\text { lecer el papel integral del sector privado en el finan- } \\
\text { ciamiento y la prestación de la educación. }\end{array}$ & $\begin{array}{l}\text { Decreto } 1528 \text { del } 24 \text { de ju- } \\
\text { lio de } 2002 . \\
\text { Decreto } 1283 \text { de } 2002 .\end{array}$ & $\begin{array}{l}\text { Se posibilita la contratación con entida- } \\
\text { des privadas entregando incluso estable- } \\
\text { cimientos del Estado. } \\
\text { Privilegió a la empresa privada educativa } \\
\text { pero persiguió, desde su esquema tec- } \\
\text { nocrático de evaluación de desempeño } \\
\text { y competencias, a las instituciones y do- } \\
\text { centes públicos. }\end{array}$ \\
\hline $\begin{array}{l}\text { Mejorar la transición de la escuela al mundo adulto } \\
\text { mediante un ajuste del contenido de la educación } \\
\text { secundaria para equipar a la juventud con los conoci- } \\
\text { mientos y valores que permitan participar productiva- } \\
\text { mente en el trabajo y la sociedad, y asistir a la región } \\
\text { para que cumpla con la meta de la Segunda Cumbre } \\
\text { de un } 75 \text { por ciento de alcance en el nivel secundario. }\end{array}$ & $\begin{array}{l}\text { Plan Nacional de Desarro- } \\
\text { Ilo (2010-2014). }\end{array}$ & $\begin{array}{l}\text { Planteó alianzas estratégicas entre insti- } \\
\text { tuciones de educación superior, institu- } \\
\text { ciones con programas de educación me- } \\
\text { dia técnica, sector productivo, gobiernos } \\
\text { regionales y locales, SENA, y centros de } \\
\text { innovación, productividad y desarrollo } \\
\text { tecnológico, con el fin de ampliar la ofer- } \\
\text { ta y crecer en cobertura. } \\
\text { En el caso del SENA, los programas abier- } \\
\text { tos se han realizado sobre la base de ci- } \\
\text { clos disciplinares dirigidos al desarrollo } \\
\text { de competencias y acordes con los re- } \\
\text { querimientos del sector productivo. }\end{array}$ \\
\hline $\begin{array}{l}\text { Elevar la calidad pedagógica y revitalizar las escuelas } \\
\text { públicas para alumnos pobres al apoyar mejoras en la } \\
\text { enseñanza y el aprendizaje, tales como mejoras radi- } \\
\text { cales en la capacitación de maestros, la introducción } \\
\text { de evaluaciones de escuelas y maestros, proveer a } \\
\text { los maestros de herramientas eficaces de evaluación } \\
\text { estudiantil para diagnosticar el rendimiento, y crear } \\
\text { incentivos para un desarrollo profesional significativo } \\
\text { de los maestros. }\end{array}$ & Decreto No. 3433 de 2008. & $\begin{array}{l}\text { Reglamenta la expedición de licencias } \\
\text { de funcionamiento de los establecimien- } \\
\text { tos no oficiales interesados en prestar } \\
\text { el servicio educativo. Con esto se busca } \\
\text { ejercer un mayor control sobre la tota- } \\
\text { lidad de los establecimientos educativos } \\
\text { (oficiales y no oficiales), contribuyendo } \\
\text { de esta manera "al mejoramiento de la } \\
\text { calidad educativa vía un mayor control } \\
\text { de las condiciones iniciales, de enseñan- } \\
\text { za y de requisitos de los establecimientos } \\
\text { educativos no oficiales". }\end{array}$ \\
\hline
\end{tabular}




\begin{tabular}{|c|c|c|}
\hline RECOMENDACIÓN DEL BANCO MUNDIAL & LEY/ DECRETO/ PLAN & EFECTOS DE SU APLICACIÓN \\
\hline $\begin{array}{l}\text { Para que las nuevas democracias de la región pros- } \\
\text { peren, deben contar con la amplia participación de } \\
\text { una ciudadanía educada e informada }\end{array}$ & \begin{tabular}{|l} 
Plan Nacional de \\
Desarrollo (2010-2014)
\end{tabular} & $\begin{array}{l}\text { Este plan define la educación como el } \\
\text { "instrumento más poderoso para reducir } \\
\text { la pobreza y el camino más efectivo para } \\
\text { alcanzar la prosperidad" }\end{array}$ \\
\hline $\begin{array}{l}\text { Los rápidos cambios económicos y tecnológicos es- } \\
\text { tán exigiendo que los gobiernos de la región invier- } \\
\text { tan en las habilidades y la educación de la fuerza } \\
\text { laboral. }\end{array}$ & $\begin{array}{l}\text { "Bases del Plan Nacional } \\
\text { de Desarrollo 2002-2006". } \\
\text { "Plan Nacional de Desa- } \\
\text { rrollo (2010-2014). } \\
\text { Ley } 1286 \text { del } 23 \text { de Enero } \\
\text { de } 2009 .\end{array}$ & $\begin{array}{l}\text { Se definió la educación como "factor } \\
\text { esencial del desarrollo humano, social y } \\
\text { económico y un instrumento fundamen- } \\
\text { tal para la construcción de equidad social" } \\
\text { La "adquisición de conocimientos, com- } \\
\text { petencias y habilidades productivas" es } \\
\text { requisito para "contribuir al crecimiento e } \\
\text { incrementar la productividad del empleo". } \\
\text { La nueva ley tiene como pretensión trans- } \\
\text { formar a Colciencias en Departamento Ad- } \\
\text { ministrativo y fortalecer el Sistema Nacio- } \\
\text { nal de Ciencia, Tecnología e Innovación en } \\
\text { Colombia, para lograr un modelo producti- } \\
\text { vo," sustentado en la ciencia, la tecnología } \\
\text { y la innovación, para darle valor agregado } \\
\text { a los productos y servicios de nuestra eco- } \\
\text { nomía y propiciar el desarrollo productivo } \\
\text { y una nueva industria nacional. }\end{array}$ \\
\hline
\end{tabular}

\section{CONCLUSIONES}

Esta reconstrucción histórica del proceso de intervención del BM en la educación colombiana permite identificar siete grandes conclusiones:

1. A medida que se acerca el más importante proceso de reforma a la educación en Colombia referida a la promulgación de la nueva Ley General de Educación 115 del 94, los créditos asumidos por el país frente al BM se incrementan en un 125\% aproximadamente. Esto genera las condiciones propicias para que el BM se posicione como un actor en la lucha de fuerzas que significó esta reforma.

2. Los créditos asumidos por la nación colombiana entre 1992 y 1996 se enfocaron en la educación primaria y secundaria, entre 1998 y 2002 los créditos se dirigen principalmente a reformas para educación técnica y superior. Esto significa que las orientaciones del BM asumidas por Colombia afectaron directamente a una generación escolar que hoy en día forma parte de una emergente clase profesional. El proyecto de BM de reforma se ejecutó en un plazo de 15 años, lo suficiente para formar una nueva generación de colombianos adeptos al discurso de las competencias. 
3. La intervención del BM condujo a la implementación de reformas administrativas y distribución de los recursos que en teoría hacían al sistema educativo más eficiente. En la práctica ha fomentado la corrupción a nivel de los gobiernos locales y ha profundizado las brechas de desarrollo entre las ciudades de primer orden y las zonas rurales.

4. La reforma al sistema de investigación nacional ha eliminado del horizonte académico a las humanidades y las ciencias sociales, imponiendo una visión del desarrollo y la innovación en los términos de una economía extractiva incapaz de crear Ciencia para el servicio de la sociedad.

5. Las apuestas en materia de Educación Superior se reducen la formación Técnica y Tecnológica cerrando paulatinamente el acceso a la educación profesional y posgradual.

La única institución que parece favorecerse de esta situación es el Servicio Nacional de Aprendizaje SENA, principal referente en Latinoamérica y el Caribe en formación de competencias técnicas laborales.

6. La dependencia económica en este renglón de la economía opera de modo similar a oros sectores: Las recomendaciones del BM limitan la posibilidad de generar estrategias para acrecentar la comunidad científica nacional, mientras que la masa de trabajadores altamente cualificados para labores operarias crece exponencialmente.

7. La concepción de la educación como un instrumento para, en detrimento de la definición de la educación como derecho fundamental, ha trasladado la responsabilidad de la misma de manos del Estado a un sinfín de organizaciones privadas y del tercer sector. Las posibilidades de revertir este proceso desde las instancias del gobierno nacional se reducen al mínimo, en la medida que organismos como el BM adquieran un mayor grado de injerencia en la educación por su permanencia y por la relación de dependencia generada en países como Colombia.

\section{REFERENCIAS BIBLIOGRÁFICAS}

Atehortúa, Adolfo. (2006): "La Revolución Educativa, transcurso, resultados y perspectiva”. En, Análisis político. Bogotá: IEPRI, Universidad Nacional de Colombia. No. 57.

(2012): "El Banco Mundial y las Políticas Educativas en Colombia”. (Ponencia) En, Simposio Internacional de pedagogía-Humanidades y Educación. Universidad Autónoma de Oc- 
cidente. Cali-Colombia. 93N+EDUCATIVA\%22\%3A+TRANSCURSO\%2C+RESULTADOS+Y + PERSPECTIVA+pdf

Banco Mundial (2000): La Educación en América Latina y el Caribe. Informe preliminar.

Bocanegra, Henry (s.f.p): Las Políticas Educativas y el Magisterio Colombiano en la Década de los 80'. Universidad Libre. Bogotá, D.C.

Departamento Nacional de Planeación (2002): Bases del Plan Nacional de Desarrollo 2002-2006. Hacia un Estado Comunitario. Bogotá: Presidencia de la República.

(2007): Plan Nacional de Desarrollo 2006-2010. Estado comunitario: desarrollo para todos. Bogotá, Presidencia de la República. Tomo I.

(2011): Plan Nacional de Desarrollo 2010-2014. Prosperidad para todos. Bogotá, Presidencia de la República.

Gonzalés, William (2012): "Que Universidad queremos”. Facultad de Humanidades. Universidad del Valle.

Ramirez, María T. y TELLEZ, Juana P. (2006): La educación primaria y secundaria en Colombia en el siglo XX.

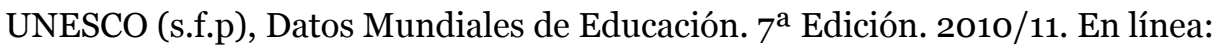
http://www.ibe.unesco.org/es/

http://www.ibe.unesco.org/es/servicios/documentos-en-linea/datos-mundiales-deeducacion/septima-edicion-2010-11.html

Vasco, Carlos Eduardo (2003): Objetivos Específicos, Indicadores de logro y competencias: ¿y ahora estándares? Fecode. Educación y Cultura: Revista trimestral del Centro de Estudios e Investigaciones de la Federación Colombiana de Educadores. No 62.Bogotá.

World Bank, (1999): Educational change in Latin America and the Caribbean. World Bank, Human Development Network. En línea:

http://www.fmmeducacion.com.ar/Sisteduc/Banco/bmeducamerica.pdf 


\section{CURRICULUM VITAE}

\section{Guillermo Andrés Duque Silva}

Licenciado en Historia de la Universidad del Valle.

Especialista en Gerencia de Instituciones Educativas de la Universidad del Tolima.

Magister (c) en Políticas Públicas e la Universidad del Valle.

Magister (c) en Filosofía de la Universidad del Valle.

Docente catedrático Universidad del Cauca Facultad de Derecho, sede Norte.

Coordinador Centro de investigaciones Socio-jurídicas Universidad Cooperativa de Colombia. Asesor de monografía de grado "El grave error de la formación de competencias en Colombia" de la estudiante Yuly Andrea García Castillo del programa de Licenciatura en Ciencias Sociales de la Universidad del Valle.

\section{fielalatierra@hotmail.com}

\section{Yuly Andrea García}

Estudiante de último semestre del programa de Licenciatura en Ciencias Sociales de la Universidad del Valle.

Docente del Colegio Waldorf Luis Horacio Gómez.

yuligarciao1@gmail.com 